\title{
Influence d'acides organiques sur la désorption de l'américium 241 et du neptunium 237 à partir d'un sol cultivé acide
}

\author{
S. ROUSSEL-DEBET*, C. COLLE*, P. HURTEVENT*, M. MORELLO*
}

(Manuscrit reçu le 14 février 2000, révisé le 30 mai 2000, accepté le 23 aoùt 2000)

RÉSUMÉ La matière organique affecte le comportement des actinides transuraniens dans l'environnement, notamment dans les sols. Cette étude expérimentale vise à évaluer si des acides carboxyliques (citrique, lactique, oxalique, vanillique), naturellement présents dans la rhizosphère d'un sol agricole ou sous litière de sol forestier, modifient la disponibilité de ${ }^{241} \mathrm{Am}$ ou ${ }^{237} \mathrm{~Np}$. Les résultats obtenus montrent que ces acides augmentent la désorption de l'américium et du neptunium vers la solution du sol, avec un effet particulièrement marqué de l'acide citrique. La fraction désorbée est multipliée par plus de 10 pour l'acide citrique $0,1 \mathrm{M}$, et par 3 ou 4 pour les autres acides, en relation avec la complexation des radionucléides par ces acides. L'augmentation de la désorption s'accrô̂t avec la concentration molaire des acides, du fait de la baisse du pH de la solution de sol acidifié, sauf pour l'acide oxalique. Les conditions d'humidité du sol semblent affecter l'intensité de la désorption: le pourcentage désorbé est approximativement 10 fois moindre pour des expériences en «batches (extractions liquide-solide, en conditions saturées) que lors d'extractions par centrifugation, correspondant à un état hydrique insaturé du sol. Ces résultats préliminaires sont essentiellement qualitatifs, toutefois, ils montrent l'intérêt de prendre en compte des conditions physico-chimiques plus réalistes pour la détermination des coefficients de distribution des sols cultivés.

ABSTRACT Effect of organic acids on 241 americium and 237 neptunium desorption from an acidic cultivated soil.

Naturally occurring organic substances have been recognised as one important factor affecting the behaviour in the environment of transuranic actinides. This experimental study aims at evaluating if some naturally occurring carboxylic acids (vanillic, citric, lactic, oxalic) would affect ${ }^{241} \mathrm{Am}$ or ${ }^{237} \mathrm{~Np}$ availability in an acidic agricultural soil. Data show that these acids enhance the desorption of americium and neptunium from the soil, with a noticeable effect of citric acid. The fraction desorbed is multiplied by more than 10 for $0.1 \mathrm{M}$ citric acid, and by 3 or 4 with other acids, in relation with the complexation of radionuclides with these acids. The desorption increases with molar concentration of the acids because of the decrease of $\mathrm{pH}$ in acidified soils, except for oxalic acid. The soil moisture conditions seem to affect the desorption results: the percentage desorbed is approximately 10 times lower for batch experiments (saturated conditions) than for centrifugation corresponding to unsaturated soil conditions. These preliminary data

\footnotetext{
Institut de protection et de sûreté nucléaire, DPRE/SERLAB/LRE, C.E. Cadarache, BP 1, 13108 Saint-Paul-LezDurance Cedex, France.
} 
are mainly qualitative, but they show that it could be interesting to take into account more realistic physico-chemical conditions when measuring desorption ratios for cultivated soils.

\section{Introduction}

Prévoir l'évolution à long terme des actinides transuraniens est un enjeu important du fait de leur présence à chaque étape du cycle du combustible et de la persistance de leurs isotopes à vie longue dans les déchets nucléaires. Or, en dépit de nombreux travaux, la connaissance sur leur comportement et leur biodisponibilité à long terme dans le système sol-plante reste parcellaire.

Les phénomènes de sorption-désorption des actinides transuraniens dans les sols et, surtout, les roches mères, ont été très étudiés dans une perspective d'évaluation d'impact des stockages profonds (Humel et al., 2000; Kim et Marquardt, 1999; Moulin et Moulin, 1995 ; Degueldre et al., 1994). Dans le sol, le neptunium se trouverait normalement sous forme pentavalente $\mathrm{Np}(\mathrm{V}) \mathrm{O}_{2}{ }^{+}$, mais pourrait être réduit à l'état $\mathrm{IV}$, (en $\mathrm{Np}(\mathrm{OH})_{4}$ ou $\left.\mathrm{Np}(\mathrm{OH})_{3}{ }^{+}\right)$notamment en milieu anaérobie, ou en présence de fer ou par la matière organique du sol, alors que l'état le plus stable de l'américium serait la forme trivalente $\mathrm{Am}^{3+}$ (Silva et Nitsche, 1995).

La forte réactivité chimique des transuraniens se traduit par des phénomènes antagonistes avec, d'une part, la formation de complexes (notamment avec les carbonates) et de pseudo-colloïdes susceptibles de migrer et, d'autre part, l'hydrolyse en composés insolubles, l'adsorption sur les particules argileuses et la formation de complexes plus ou moins solubles avec les molécules organiques. Les interactions avec les colloides jouent un rôle important: l'étude de la vitesse de migration du neptunium sur un sable met en évidence une augmentation de la solubilité apparente de $\mathrm{Np}$ (IV), liée à la formation de pseudo-colloïdes alors que la sorption de $\mathrm{Np}(\mathrm{V})$ sur les colloïdes humiques est plus faible et peut être négligée (Kim et al., 1994). La sorption de l'américium est décrite par Degueldre et al. (1994) comme une compétition entre la formation de complexes en solution avec les carbonates et d'adsorption à la surface des colloïdes: l'augmentation des ions carbonates (à partir de $10^{-2} \mathrm{M}$ ) à $\mathrm{pH} 8$ réduit assez sensiblement l'adsorption de l'américium sur les colloïdes. Pour le neptunium, la solubilité décroît quand le $\mathrm{pH}$ augmente, en liaison avec la complexation de Np (V) avec les carbonates (Nitsche, 1993). Le neptunium se fixerait préférentiellement sur la surface externe des argiles (Askarieh et al., 1993) et de ce fait, sa sorption ne serait pas irréversible; les processus d'adsorption et de désorption semblent contrôlés par la surface spécifique des argiles et par le pH. À partir d'un $\mathrm{pH}$ voisin de 7, l'extractabilité diminue avec l'augmentation de $\mathrm{pH}$ - qui favorise l'hydrolyse, la complexation avec les ions carbonates et la formation de colloïdes -, puis augmente vers $\mathrm{pH} 8,5$ (Nishita et al., 1979). Les hydroxydes de fer, aluminium et manganèse semblent jouer un rôle dans la 
TABLEAU I

Constantes de stabilité des complexes d'Am(III) et de $\mathrm{Np}(\mathrm{V})$ avec les substances organiques. Stability constants of Am(III) or Np (V) complexes with organics.

(Selon Kim et al., 1997; Yong et Macaskie, 1997; Carlsen, 1989).

\begin{tabular}{|c|c|c|c|}
\hline \multirow{2}{*}{ Ligand } & \multirow{2}{*}{$\frac{A m(I I I)}{\log \beta}$} & \multicolumn{2}{|c|}{$\mathrm{Np}(\mathrm{V})$} \\
\hline & & $\log \beta_{1}$ & $\log \beta_{2}$ \\
\hline Acide humique & 6,4 à 15,7 selon le $\mathrm{pH}$ & $3,3-3,8$ & \\
\hline Acide fulvique & $6,0-6,2$ & & \\
\hline Acide acétique & 1,99 & 1,33 & \\
\hline Acide citrique & 7,74 & $3,67-4,87$ & $8,45-9,05$ \\
\hline EDTA & 18,16 & $6,87-7,12$ & \\
\hline DTPA & 22,29 & & \\
\hline NTA & 11,5 & 6,80 & \\
\hline Oxalate & & $3.42-4,39$ & $5,66-7,36$ \\
\hline
\end{tabular}

EDTA : acide éthylène-diamine tétra-acétique, DTPA : acide diéthylène-triamine-penta-acétique, NTA: acide nitrilo-tri-acétique.

sorption des transuraniens, à rapprocher de l'action des sidérophores d'origine microbienne - qui permettent normalement la solubilisation de Fe(III) et son transport - et qui favoriseraient la solubilisation d'oxydes insolubles (Brainard et al., 1992; Watteau et Berthelin, 1994).

Les transuraniens sont susceptibles de se complexer, par formation de sel, principalement par réaction avec les groupements carboxyliques, ou par chélation, avec les substances organiques, notamment celles présentes dans les sols, qu'il s'agisse des molécules polycondensées d'acides humiques et fulviques, ou d'acides organiques simples (Labonne-Wall et al., 1997; Marquardt et Kim, 1998; Moulin et al., 1999; Rao et Choppin, 1995). Par ailleurs, la présence d'acides humiques ou fulviques augmente notablement les constantes de stabilité apparente de l'américium avec la silice (Labonne-Wall et al., 1997) alors que l'adsorption du neptunium sur $\mathrm{SiO}_{2}$ ne semble pas affectée par la présence ou l'absence d'acides fulviques ou d'acides aminés (Kung ct Triay, 1994). Rao et Choppin (1995) ont montré que les constantes de formation des complexes avec le neptunium sont plus faibles pour des fractions d'acides humiques de plus petites tailles, du fait que leur acidité est plus forte et que le rapport entre les sites de surface et les sites internes est plus grand, favorisant des liaisons plus solides avec des acides humiques de poids moléculaires plus grands.

Les constantes de stabilité des complexes avec les substances organiques sont élevées (Tab. I). La stabilité des complexes actinides - acides humiques diminue avec l'augmentation de la force ionique, comme pour d'autres métaux, sans doute à cause des différentes réactions de complexations compétitives qui se mettent en place avec les autres ions (Carlsen, 1989). Une diminution de $\mathrm{pH}$ a le même effet, sachant 
que l'anion (ligand) se protonise et n'est donc plus disponible: pour des $\mathrm{pH}$ allant de 4 à 6 , le logarithme de la constante de stabilité augmente linéairement avec le $\mathrm{pH}$ de 1,5 à 2 unités logarithmiques par unité de $\mathrm{pH}$.

Dans les sols, le coefficient de distribution de l'américium et du neptunium est partiellement contrôlé par les substances humiques (Tanaka et al., 1997; Tipping et al., 1995); il semblerait que la présence de matière organique soluble, non liée aux argiles, puisse favoriser la migration des actinides (Olofsson et Allard, 1983). Dans la couche supérieure du sol, les exsudats racinaires, l'activité microbienne et le lessivage des litières produisent des composés organiques, en particulier des acides solubles de faible masse moléculaire (Dinkelaker et al., 1989; Birch et Bachofen, 1990). Ces acides - oxalique, citrique, tartrique, malique, lactique, succinique, fumarique, vanillique - joueraient un rôle majeur dans la dissolution des métaux et favoriseraient leur incorporation racinaire, en particulier dans le cas du fer (Bruckert, 1970; Watteau et Berthelin, 1994). Ces acides carboxyliques pourraient donc également favoriser la solubilisation des radionucléides et augmenter leur aptitude au transfert racinaire ou à la migration. Les concentrations dans la solution du sol de ces acides sont encore mal connues. Elles pourraient atteindre un maximum de $2 \times 10^{-2} \mathrm{M}$ et leurs valeurs moyennes fluctueraient selon les conditions saisonnières autour de $1 \times 10^{-3} \mathrm{M}$ à $4 \times 10^{-3} \mathrm{M}$ (Lefèbre-Drouet et al., 1993; Keague et al., 1986). La concentration de ces composés organiques est influencée par l'activité des racines qui les sécrètent, par la présence ou l'absence d'une litière de feuilles en surface du sol - susceptible d'être lessivée par la pluie - par l'activité biologique et microbiologique du sol au voisinage de la rhizosphère, ainsi que par la saison, qui intervient aussi dans leur cycle de minéralisation.

On peut donc penser que la présence d'acides organiques simples au niveau de la zone racinaire joue un rôle dans l'aptitude des actinides transuraniens à être mobilisés, puis entraînés par lessivage vertical ou incorporés par les végétaux via l'absorption racinaire. Pour tenter de le vérifier nous avons réalisés les essais expérimentaux présentés ci-après, en étudiant l'influence de l'apport d'acides carboxyliques sur la désorption des radionucléides à partir d'un sol artificiellement contaminé en américium et neptunium. Des tests complémentaires ont également été réalisés afin de savoir si ces acides pouvaient induire une modification du transfert au végétal.

\section{Matériel et méthodes}

Le sol utilisé est un sol agricole sablonneux, faiblement acide $\left(\mathrm{pH}_{\text {eau }}=5,6\right)$ provenant de la région de La Hague (France), cf. tableau П. Deux lots de ce sol, étudié antérieurement (Cawse et Colle, 1989; Roussel-Debet et al., 1997) avaient été contaminés de façon homogène en 1985, à raison respectivement de $18,5 \pm 0,2 \mathrm{~Bq} \mathrm{~g}^{-1}(\mathrm{sec})$ $\mathrm{d}^{241} \mathrm{Am}$ et $7,4 \pm 0,06 \mathrm{~Bq} \mathrm{~g}^{-1}(\mathrm{sec}) \mathrm{de}^{237} \mathrm{~Np}$. Les acides organiques retenus sont l'acide 


\section{DÉSORPTION DE L ${ }^{241}$ Am ET DU ${ }^{237} \mathrm{~Np}$}

\section{TABLEAU II}

Analyse physico-chimique du sol (valeurs relatives au sol sec, fraction tamisée à $\mathbf{2} \mathbf{~ m m}$ ).

Physico-chemical analysis of the soil (all values are related to the dry sieved

( $<2 \mathrm{~mm}$ fraction) soil).

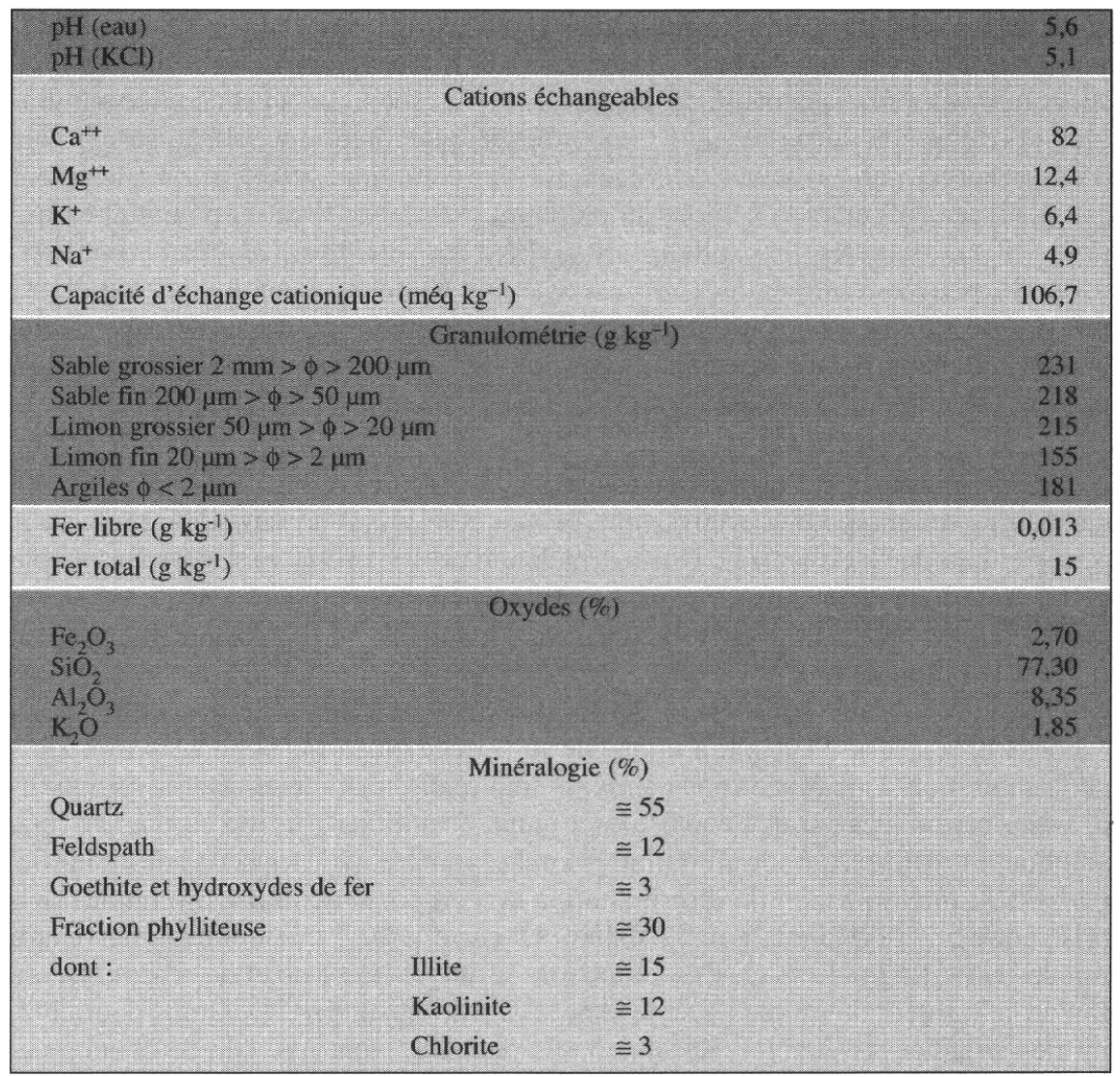

citrique $\mathrm{C}_{2} \mathrm{H}_{2} \mathrm{O}_{4}, 2 \mathrm{H}_{2} \mathrm{O}$, oxalique $\mathrm{C}_{6} \mathrm{H}_{8} \mathrm{O}_{7}, \mathrm{H}_{2} \mathrm{O}$, lactique $\mathrm{C}_{3} \mathrm{H}_{6} \mathrm{O}_{3}$ ainsi que l'acide vanillique $\mathrm{C}_{8} \mathrm{H}_{8} \mathrm{O}_{4}$, dans une gamme de concentrations variant de $0,1 \mathrm{M}$ à $0,001 \mathrm{M}$.

Des essais classiques de désorption en milieu saturé, dits «batch», ont consisté à mélanger $10 \mathrm{~g}$ de sol sec et $50 \mathrm{ml}$ de l'une des solutions d'acides organiques. Les échantillons (5 répétitions) ont été agités manuellement chaque heure durant 8 heures, puis, après 36 heures de sédimentation, $35 \mathrm{ml}$ de surnageant ont été prélevés. Des percolations ont aussi été réalisées pour l'américium avec les acides de concentration $10^{-3} \mathrm{M}: 50 \mathrm{~g}$ de sol ont été disposés dans un buchner muni d'un 
filtre cellulosique de porosité $0,45 \mu \mathrm{m}$ et $200 \mathrm{~cm}^{3}$ de chacune des solutions d'acides ont été mises à percoler durant 2 heures. Des échantillons témoins ont été réalisés en remplaçant les solutions d'acides organiques par de l'eau osmosée. Une deuxième série expérimentale a été effectuée en milieu non saturé. La solution du sol a été extraite par centrifugation. Ce type d'extraction est assimilé à la représentation d'un $p F$ compris entre 3 et 4 , c'est-à-dire aux conditions d'humidité du sol comprises entre la capacité au champ (l'eau excédentaire s'est écoulée par gravité) et le point de flétrissement (limite inférieure de rétention de l'eau susceptible d'être absorbée par les racines des végétaux). Par définition, $p F=\log _{10}(\Psi)$, le potentiel hydrique $(\Psi)$ étant la somme du potentiel gravitaire $\left(\Psi_{\mathrm{g}}\right)$, du potentiel osmotique $\left(\Psi_{0}\right)$ et du potentiel capillaire $\left(\Psi_{\mathrm{m}}\right)$. Pour les sols, hors cas très particuliers, $\Psi_{\mathrm{g}}$ et $\Psi_{0}$ peuvent être négligés. $\Psi_{\mathrm{m}}$ est égal à la hauteur à laquelle on peut élever une masse d'eau de 1 gramme, contre la pesanteur et de façon réversible. Numériquement, il est équivalent d'exprimer $\Psi_{\mathrm{m}}$ par cette hauteur d'eau $(\mathrm{cm})$ ou par l'accélération $\gamma$ exprimée en $g\left(\sim 9,81 \mathrm{~m} \mathrm{~s}^{-2}\right)$, soit $p F^{a} \log _{10}(\gamma)$.

Trente grammes de sol sec sont mélangés aux solutions d'acides organiques, en ajustant le volume pour se trouver initialement à la limite de saturation du sol, i.e. $0,53 \mathrm{~g}$ d'eau par $\mathrm{g}$ de sol sec. Chaque mélange est répété six fois, dans un double godet en téflon, permettant l'extraction de la solution du sol par centrifugation, en appliquant des accélérations croissantes de 1000 à $7500 \times \mathrm{g}$. Chaque extraction à une accélération donnée est répétée 2 fois, durant 1 heure. Le $\mathrm{pH}$ des surnageants de chaque échantillon est mesuré. En fin d'extraction, l'humidité résiduelle du sol est de $0,13 \pm 0,01 \mathrm{~g}$ d'eau par gramme de sol sec, ce qui correspond à environ $25 \%$ de la saturation. Des échantillons témoins sont réalisés en remplaçant les solutions d'acides organiques par de l'eau osmosée; une séparation (trois répétitions) de cette solution est réalisée, par ultrafiltration frontale sur membrane triacétate de cellulose, permettant théoriquement la discrimination de composés de masse atomique comprise entre 5 et 500 kilodaltons (le dalton, $\mathrm{D}$, correspond à une unité de masse atomique: $\left.1 \mathrm{D} \sim 1,67 \times 10^{-27} \mathrm{~g}\right)$. Ces extractions n'ont pas fait l'objet de répétitions, du fait de la lourdeur du protocole et de la nécessité de regrouper les extraits pour obtenir des volumes mesurables. Sur une partie du sol n'ayant pas subi de contamination par les radionucléides, la teneur en cations majeurs des solutions de sol extraites par l'eau osmosée et par l'acide citrique 0,1 M est mesurée par ICP-OES (Induced Coupled Plasma Optical Spectra Emission). Le pH des solutions de chaque échantillon est mesuré en fin d'expérimentation et la force ionique est, estimée.

Par ailleurs, des mesures du coefficient de transfert racinaire de ${ }^{241}$ Am sont effectuées avec des plantules de blé (variété Soisson). Ces essais consistent à mesurer l'activité de la partie aérienne des végétaux, d'une part, après culture sur sol contaminé et d'autre part après la contamination directe des racines avec une solution de sol extraite, en présence ou absence d'acides organiques. À cet effet, six lots de blé sont semés. Le premier lot est cultivé durant 14 jours sur le sol contaminé en ${ }^{241} \mathrm{Am}$. Le blé des cinq autres lots est semé puis cultivé durant 14 jours sur du 
sable avec une solution nutritive. Ensuite, les plantules entières sont extraites de ce support inerte et leurs racines sont rincées puis chaque lot est placé durant 24 heures dans chacune des solutions extraites du sol contaminé. Après ce délai les parties aériennes sont récoltées et traitées en vue de mesurer leur activité. Cinq répétitions comportant chacune $10 \mathrm{~g}$ de grains de blé sont effectuées pour les six lots.

Tous les prélèvements sont traités par minéralisation en milieu nitrique. Les échantillons sont mesurés par spectrométrie gamma. Si nécessaire, $1^{241}$ Am est ensuite mesuré par spectrométrie alpha après radiochimie et électrodéposition selon un protocole, dérivé de celui décrit par Morello et al. (1986), schématisé figure 1.

\section{Résultats et discussion}

Les résultats, obtenus pour les sols et présentés au tableau III sont exprimés sous forme d'un coefficient de désorption $K\left(\mathrm{~cm}^{3} \mathrm{~g}^{-1}\right)$ égal au rapport entre l'activité massique initiale du sol $\left(\mathrm{Bq} \mathrm{g}^{-1}\right)$ et l'activité volumique de la solution extraite $\left(\mathrm{Bq} \mathrm{cm}{ }^{-3}\right)$. Ce coefficient $K$ est proche du coefficient de distribution $(K \mathrm{~d})$ sans lui être rigoureusement égal : $K$ d est égal au rapport de l'activité massique finale du sol à l'activité volumique de la solution extraite et sa détermination s'effectue habituellement après avoir vérifié que la sorption-désorption du contaminant obéit à un équilibre chimique réversible et indépendant.

La désorption du ${ }^{237} \mathrm{~Np}$ est toujours notablement plus élevée que celle de $1^{1241} \mathrm{Am}$, ce qui n'est pas surprenant. En milieu saturé ( «batch»), le coefficient $K$ des échantillons témoins est de l'ordre de $4300 \mathrm{~cm}^{3} \mathrm{~g}^{-1}$ pour l' ${ }^{241} \mathrm{Am}$ et de $140 \mathrm{~cm}^{3} \mathrm{~g}^{-1}$ pour le ${ }^{237} \mathrm{~Np}$. Ces valeurs sont du même ordre de grandeur que les moyennes respectives, pour l'américium et le neptunium, des $K \mathrm{~d}$ sur sols acides (Thibault et al., 1990) et sont en accord avec les observations montrant une mobilité supérieure du neptunium par rapport à l'américium (Hursthouse et Livens, 1993). Le coefficient $K$ est, en outre, notablement plus faible pour les extractions en milieu insaturé : de l'ordre de 700 à $800 \mathrm{~cm}^{3} \mathrm{~g}^{-1}$ pour $\mathrm{l}^{\text {,241 }} \mathrm{Am}$ et 22 à 34 pour le ${ }^{237} \mathrm{~Np}$, traduisant une tendance accrue à la désorption avec la dessiccation du sol. Ce fait a déjà été observé dans le cas de l'américium, sur un sol calcaire, et du césium, sur différents sols (Roussel-Debet et al., 1997). Cet aspect pose la question des conditions de détermination du coefficient de distribution qui est habituellement mesuré avec un rapport entre volume de solution et masse de sol de l'ordre de 5 à 10 et dont la valeur serait surestimée pour les conditions hydriques habituelles du sol. Des expériences complémentaires devraient éclaircir ce point.

L'ajout d'acides organiques au sol se traduit par une augmentation - plus ou moins marquée - de la désorption, pour la plupart des conditions expérimentales, avec un effet particulièrement sensible de l'acide citrique, qui est, par ailleurs, 


\section{1 - Echantillon} minéralisé

$+10 \mathrm{~cm}^{3} \mathrm{HCl} 9 \mathrm{M}$

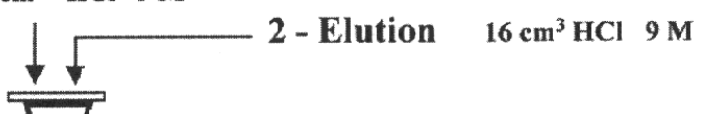

\section{Résine cationique \\ 2 g AGIX50 WX8 200 - 400 mesli \\ Fixation de U, Pu}

\section{Résine anionique}

6,5 g AGIX8 100 - 200 mesli

Fixation de Fe (III)

\section{3 - Eluat ( $\mathrm{Ni}, \mathrm{Cu}$, lanthanids $+\mathrm{Am}, \mathrm{Cm}$ )}

4 - Rinçage $2 \times 5 \mathrm{~cm}^{3} \mathrm{HNO}_{3} \quad 2 \mathrm{M}+5 \mathrm{~cm}^{3} \mathrm{Al}\left(\mathrm{NO}_{3}\right)_{3} \quad 1 \mathrm{M}$ (Am retenu)

5 - Elution de $\mathrm{Am} \quad 12 \mathrm{~cm}^{3} \mathrm{HNO}_{3} \quad 0,05 \mathrm{M}$

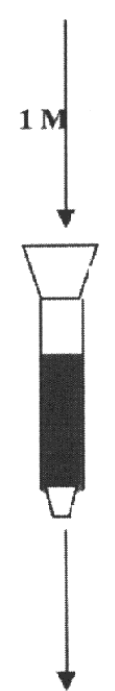

résine TRU-spec

$50-100 \mu$

5 - Eluat (Am)

6 - Electrodéposition

7 - Spectrométrie $\alpha$

Fig. 1. -Séparation radiochimique de $l^{, 241} \mathrm{Am}$.

Radiochemical separation of ${ }^{241} A m$. 


\section{TABLEAU III}

Coefficient de désorption $\mathrm{K}\left(\mathrm{cm}^{3} \mathrm{~g}^{-1}\right)$.

Desorption coefficient $\mathrm{K}\left(\mathrm{cm}^{3} \mathrm{~g}^{-1}\right)$.

a) Conditions saturées (saturated conditions).

\begin{tabular}{|c|c|c|c|c|}
\hline${ }^{237} \mathrm{~Np}$ batch & & ${ }^{21}$ Am batch & Am percolatio & \\
\hline témoin & & $142 \pm 39$ & $4260 \pm 561$ & $1504 \pm 122$ \\
\hline acide citrique & $\begin{array}{l}0,001 \mathrm{M} \\
0,01 \mathrm{M} \\
0,1 \mathrm{M}\end{array}$ & $\begin{array}{l}23 \pm 2 \\
12 \pm 1 \\
10 \pm 1\end{array}$ & $\begin{array}{c}1290 \pm 128 \\
243 \pm 27 \\
65 \pm 6\end{array}$ & $907 \pm 89$ \\
\hline acide oxalique & $\begin{array}{l}0,001 \mathrm{M} \\
0,01 \mathrm{M} \\
0,1 \mathrm{M}\end{array}$ & $\begin{array}{l}35 \pm 4 \\
44 \pm 5 \\
42 \pm 4\end{array}$ & $\begin{array}{l}1998 \pm 185 \\
1986 \pm 183 \\
1551 \pm 223\end{array}$ & $2033 \pm 223$ \\
\hline acide lactique & $\begin{array}{l}0,001 \mathrm{M} \\
0,01 \mathrm{M} \\
0,1 \mathrm{M}\end{array}$ & $\begin{array}{c}201 \pm 71 \\
59 \pm 9 \\
32 \pm 3\end{array}$ & $\begin{array}{c}3174 \pm 255 \\
1799 \pm 150 \\
256 \pm 15\end{array}$ & $1164 \pm 88$ \\
\hline acide vanillique & $\begin{array}{l}0,001 \mathrm{M} \\
0,01 \mathrm{M}\end{array}$ & $\begin{array}{l}40 \pm 4 \\
34 \pm 4\end{array}$ & $\begin{array}{l}2676 \pm 268 \\
1789 \pm 179\end{array}$ & $954 \pm 54$ \\
\hline
\end{tabular}

Moyennes arithmétiques ( $\pm 2 \times$ écart-type) de 5 répétitions. (Arithmetic means $( \pm 2 \times$ standard deviation) of five replicates.)

b) Extraction de la solution du sol par centrifugation (soil solution extracted by centifugation).

\begin{tabular}{|lccc|}
\hline & Accélération & ${ }^{237} \mathrm{~Np}$ & ${ }^{241} \mathrm{Am}$ \\
\hline témoin & $1000 \times g$ & $21,7 \pm 2,9$ & $787 \pm 73$ \\
& $2500 \times g$ & $33,6 \pm 8,4$ & $880 \pm 90$ \\
& $5000 \times g$ & non détecté & $669 \pm 83$ \\
acide citrique $0,1 \mathrm{M}$ & $7500 \times g$ & non détecté & $373 \pm 34$ \\
& $1000 \times g$ & $2,7 \pm 0,3$ & $10,0 \pm 0,4$ \\
& $2500 \times g$ & $2,5 \pm 0,2$ & $6,0 \pm 0,2$ \\
& $5000 \times g$ & $2,5 \pm 0,3$ & $7,3 \pm 0,5$ \\
acide oxalique $0,1 \mathrm{M}$ & $7500 \times g$ & $2,2 \pm 0,3$ & $9,1 \pm 1,0$ \\
& $1000 \times g$ & $6,3 \pm 0,3$ & $434 \pm 46$ \\
& $2500 \times g$ & $7,5 \pm 0,3$ & $667 \pm 66$ \\
acide lactique $0,1 \mathrm{M}$ & $5000 \times g$ & $8,0 \pm 1,0$ & $564 \pm 55$ \\
& $7500 \times g$ & $12,2 \pm 4,3$ & $593 \pm 60$ \\
& $1000 \times g$ & $8,8 \pm 0,5$ & $81 \pm 3$ \\
& $2500 \times g$ & $9,4 \pm 0,5$ & $78 \pm 3$ \\
acide vanillique $0,01 \mathrm{M}$ & $5000 \times g$ & $31,9 \pm 10,0$ & $80 \pm 6$ \\
& $7500 \times g$ & $18,5 \pm 7,4$ & $61 \pm 7$ \\
& $1000 \times g$ & $18,5 \pm 1,1$ & $848 \pm 86$ \\
& $2500 \times g$ & $20,9 \pm 1,9$ & $1038 \pm 109$ \\
& $5000 \times g$ & $13,7 \pm 1,6$ & $770 \pm 79$ \\
& $7500 \times g$ & non détecté & $727 \pm 75$ \\
\hline
\end{tabular}

Chaque valeur dérive d'une mesure sur la totalité des 6 godets ( \pm incertitude de mesurage). (Each value is derived from one measurement of six pots ( \pm dubiousness of measurement).)

un complexant reconnu des actinides. Dans les expériences «batch», en conditions saturées, la désorption est particulièrement accrue: la fraction de ${ }^{237} \mathrm{~Np}$ désorbé, égale au rapport entre l'activité totale de la solution et l'activité totale initiale 
TABLEAU IV

Caractéristiques physicochimiques des solutions extraites. Physico-chemical characteristics of extracted solutions.

a) $\mathrm{pH}$ et force ionique ( $\mathrm{pH}$ and ionic strength).

\begin{tabular}{|llcc|}
\hline & & $\mathrm{pH}$ & Force ionique (M) \\
\hline témoin & & $5,3 \pm 0,2$ & 0,03 \\
acide citrique & $0,001 \mathrm{M}$ & $4,6 \pm 0,2$ & 0,03 \\
& $0,01 \mathrm{M}$ & $3,8 \pm 0,2$ & 0,04 \\
& $0,1 \mathrm{M}$ & $2,5 \pm 0,1$ & 0,1 \\
\hline acide oxalique & $0,001 \mathrm{M}$ & $5,5 \pm 0,2$ & 0,03 \\
& $0,01 \mathrm{M}$ & $4,1 \pm 0,2$ & 0,04 \\
\hline \multirow{2}{*}{ acide lactique } & $0,1 \mathrm{M}$ & $1,5 \pm 0,1$ & 0,1 \\
& $0,001 \mathrm{M}$ & $4,9 \pm 0,2$ & 0,03 \\
& $0,01 \mathrm{M}$ & $4,7 \pm 0,2$ & 0,04 \\
\hline acide vanillique & $0,1 \mathrm{M}$ & $3,2 \pm 0,1$ & 0,1 \\
& $0,001 \mathrm{M}$ & $4,0 \pm 0,2$ & 0,03 \\
\hline
\end{tabular}

b) Concentrations en cations $\left(\mathrm{mg} \mathrm{dm}^{-3}\right)$ (Cations concentrations $\left(\mathrm{mg} \mathrm{dm}^{-3}\right)$ ).

\begin{tabular}{|c|cccccc|}
\hline & \multicolumn{3}{|c}{ Centrifugations eau osmosée } & \multicolumn{3}{c|}{ Centrifugations acide citrique $0,1 \mathrm{M}$} \\
\hline $\mathrm{Al}$ & $1000 \times g$ & $2500 \times g$ & $5000 \times g$ & $1000 \times g$ & $2500 \times g$ & $5000 \times g$ \\
$\mathrm{Fe}$ & 1,5 & 1,4 & 1,2 & 537 & 502 & 519 \\
$\mathrm{Ca}$ & 1,2 & 1,0 & 1,0 & 1695 & 1687 & 1690 \\
$\mathrm{~K}$ & 333 & 219 & 191 & 1870 & 1915 & 1777 \\
$\mathrm{Mg}$ & 62 & 54 & 42 & 99 & 91 & 105 \\
$\mathrm{Na}$ & 60 & 39 & 34 & 190 & 166 & 150 \\
\hline
\end{tabular}

du sol, qui est d'environ $3 \%$ dans les échantillons témoins, est multipliée par un facteur supérieur à 10 en présence d'acide citrique $0,1 \mathrm{M}$ et par 3 à 4 avec les autres acides et concentrations, à l'exception de l'acide lactique 0,001 M. Pour l' ${ }^{241} \mathrm{Am}$, la même tendance est observée, l'effet le plus marquant étant celui de l'acide citrique, avec 0,5 à $8 \%$ désorbés pour les concentrations 0,1 à $0,001 \mathrm{M}$. L'influence de ces acides organiques est d'autant plus marquée que leur concentration est élevée, à l'exception de l'acide oxalique, en raison de la précipitation probable des complexes entre actinides et oxalates. Les essais réalisés par percolation montrent une tendance identique, avec un coefficient de sorption moins élevé d'un facteur 1,4 à 2,8. En ce qui concerne les extractions en conditions insaturées, les résultats vont dans le même sens avec un effet encore plus net de l'acide citrique: respectivement $15 \%$ et $5 \%$ de l'activité totale du sol en ${ }^{237} \mathrm{~Np}$ et ${ }^{241} \mathrm{Am}$ sont désorbés avec la concentration $0,1 \mathrm{M}$, ce qui représente plus de 10 fois la valeur obtenue pour le témoin. L'activité volumique de chaque extrait (correspondant à une accélération donnée) est relativement constante pour chaque traitement. Le maximum d'activité est extrait lors des deux premières centrifugations à $1000 \times g$ et $2500 \times g$, du fait que cellesci extraient $90 \%$ du volume total. Cette augmentation de la désorption s'explique 
par la capacité complexante des acides carboxyliques vis-à-vis de l'américium et du neptunium et par la modification des conditions de $\mathrm{pH}$ (diminution) et de force ionique (augmentation) qui ont tendance à diminuer la stabilité des complexes et donc à favoriser le passage en solution des radionucléides (Tab. IV). En outre, l'acide citrique augmente notablement la désorption de l'aluminium et du fer : $10 \%$ des oxydes du sol sont dissous avec une concentration $0,1 \mathrm{M}$ d'acide citrique, ce qui est en accord avec les fortes constantes de stabilité des complexes citrate-Fe ${ }^{3+}$ $(\log K=11,4)$ et citrate-Al ${ }^{3+}(\log K=8,5)$. Il est possible qu'un des mécanismes impliqués soit la dissolution des radionucléides par un effet d'entraînement.

Par ailleurs, l'augmentation de la désorption en présence d'acides organiques de faible masse moléculaire s'accompagne d'une modification de la répartition des radionucléides de la solution du sol. Le fractionnement de la solution du sol obtenue par extraction en centrifugeant le sol à $2500 \times g$ indique une différence de répartition des deux radionucléides: $72 \%$ du neptunium et $53 \%$ de l'américium se trouvent dans la fraction inférieure à $5 \mathrm{kD}$. Ce résultat est en accord avec des études antérieures (Nisbet, 1993; Agapkina et Kracke, 1995) qui ont montré une corrélation entre l'importance de la biodisponibilité d'un radionucléide et l'accroissement de sa présence dans les fractions de faible masse moléculaire.

Les solutions d'acides 0,01 et $0,001 \mathrm{M}$ utilisées diminuent le $\mathrm{pH}$ de la solution du sol d'environ 1 à 1,5 unités pH par rapport aux échantillons témoins. Toutefois, il ne semble pas que ce paramètre soit uniquement à l'origine de l'augmentation de la désorption, car un effet similaire des acides organiques sur la désorption de l'américium a également été observé sur des solutions d'un sol calcaire, dont les pH n'étaient pas modifiés, grâce à l'effet tampon des carbonates du sol (RousselDebet et al., 1998). Par ailleurs, in situ, le pH de la solution du sol, à proximité de l'extrémité active des racines, peut être très acidifié, du fait de l'excrétion de protons à l'apex des racines. Aussi peut-on considérer qu'une augmentation de la solubilisation des radionucléides est susceptible de se produire, en particulier dans la zone racinaire, sous l'effet de conditions d'environnement qui favorisent la production d'acides organiques.

Les résultats obtenus en contaminant par voie racinaire des plantules de blé en ${ }^{241}$ Am avec la solution de sol témoin et du même sol lessivé par les acides organiques sont présentés figure 2. Les transferts à partir des solutions de sol acidifié sont plus faibles que ceux du témoin (solution de sol percolée à l'eau), ce qui semble indiquer qu'il n'y aurait pas de corrélation du transfert avec la désorption. Toutefois, ce résultat ne peut être affirmé avec certitude, compte tenu d'un possible artéfact lié à l'abaissement du pH de la solution dans le cas des sols acidifiés. Le coefficient de transfert entre la solution du sol et le végétal exprimé en $\mathrm{cm}_{\text {solution }}^{3} \mathrm{~g}_{\mathrm{vég} \text { ć́al sẹc, }}^{-1}$ est de $0,83 \pm 0,68$ pour $l^{1241} \mathrm{Am}$, ce qui est du même ordre de grandeur que celui obtenu, dans les mêmes conditions, pour le ${ }^{134} \mathrm{Cs}: 0,9 \pm 0,12$; en revanche, le facteur de transfert racinaire, sol $\rightarrow$ végétal, égal à $1,9 \times 10^{-4} \pm 1,6 \times 10^{-4} \mathrm{~g}_{\text {sol sec }}^{-1} \mathrm{~g}_{\text {végéćal sec }}^{-1}$ pour 
$\mathrm{Bq}^{-1}$ par Bq cm${ }^{-3}$

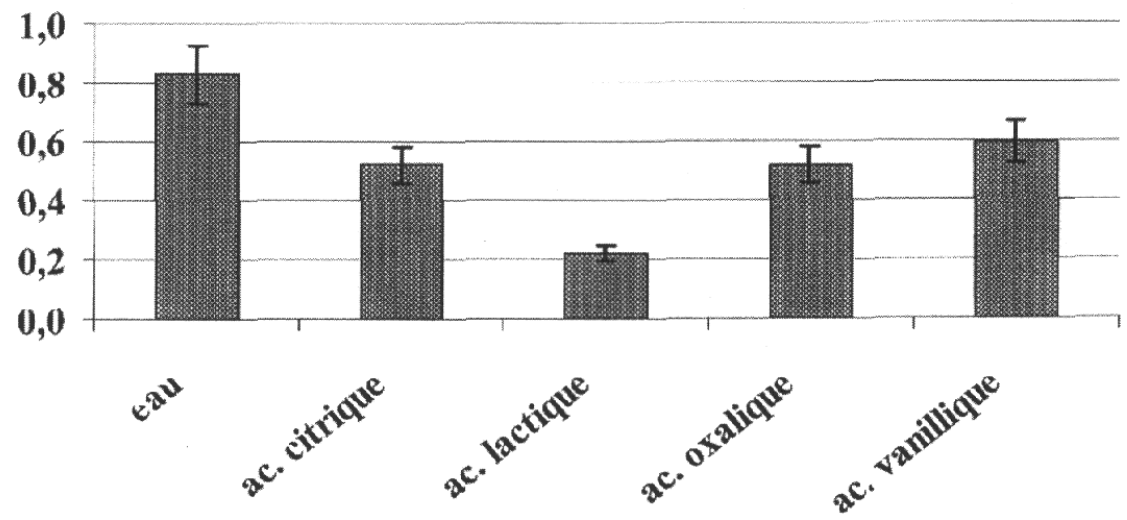

Fig. 2. - Transfert de $l^{241} \mathrm{Am}$ au blé à partir de la solution du sol obtenue par percolation avec de l'eau ou des acides organiques (0,I M, sauf acide vanillique: 0,0I M).

${ }^{241}$ Am transfer to wheat from soil solutions obtained by leaching with water or organic acids (0.1 M, except vanillic acid: $0.01 \mathrm{M}$ ).

$1^{1241}$ Am est très inférieur à celui du césium : $0,027 \pm 0,002 \mathrm{~g}_{\text {sol sec }}^{-1} \mathrm{~g}_{\text {végétal sec }}^{-1}$ (RousselDebet et al., 1997). Ces résultats préliminaires semblent indiquer que, lors du transfert racinaire, l'absorption d'un radionucléide par les racines, à partir de la solution du sol, dépend peu du type de radionucléide et que la valeur du facteur de transfert est gouvernée, en priorité, par l'intensité de la désorption du radionucléide à partir de la phase solide du sol vers la solution du sol.

\section{Conclusion}

La période des actinides transuraniens susceptibles d'être disséminés dans l'environnement est suffisamment longue pour que leur mobilité soit, à terme, influencée par des phénomènes qui interviennent dans le cycle de la matière organique. Des acides carboxyliques, normalement présents dans la rhizosphère tendent à favoriser la désorption du ${ }^{237} \mathrm{~Np}$ et de $\mathrm{l}^{\prime 241} \mathrm{Am}$ à partir de sols, sans effet immédiatement perceptible sur leur biodisponibilité. Les fluctuations naturelles de composition de la solution du sol en acides organiques pourraient donc induire des alternances d'immobilisation et de relargage de ces radionucléides. Par ailleurs, des conditions hydriques inférieures à la saturation du sol, plus réalistes qu'une classique extraction liquide-solide, semblent favoriser la désorption du ${ }^{237} \mathrm{~Np}$ et de $1^{241} \mathrm{Am}$. L'approfondissement de ce point pourrait permettre d'améliorer la 
détermination du coefficient de distribution $K \mathrm{~d}$ et aboutir à une modélisation plus fine de la migration dans les sols.

\section{RÉFÉRENCES}

Agapkina G.I., Kracke W. (1995) Association of Chernobyl derived ${ }^{239,240} \mathrm{Pu},{ }^{241} \mathrm{Am},{ }^{90} \mathrm{Sr}$ and ${ }^{137} \mathrm{Cs}$ with organic matter in the soil solution, J. Environ. Radioact. 29, 257-269.

Askarieh M.M., Hansford M.I., Staunton S., Rees S. (1993) Complexation of $N p(V)$ in aqueous solutions. CCE- EUR-14743, final report.

Birch L., Bachofen R. (1990) Complexing agents from micro-organisms, Experientia 4010, 827-834.

Brainard J.R., Strietelmeier B.A., Smith P.H. (1992) Actinide binding and solubilization by microbial siderophores, Radiochim. Acta, 58-59, 357-363.

Bruckert S. (1970) Influence des composés organiques solubles sur la pédogénèse en milieu acide, Ann. Agro. 21, 421-452 \& 725-755.

Carlsen L. (1989) CEC, The role of organics on the migration of radionuclides in the geosphere. EUR12024 final report.

Cawse P.A., Colle C. (1989) UIR, Variability in soil to plant transfer of neptunium, americium and curium. VIth report of the working group soil-to-plant, pp. 22-35. RIVM, Bilthoven.

Degueldre C., Ulrich H.J., Silby H. (1994) Sorption of ${ }^{241}$ Am onto montmorillonite illite and hematite colloids, Radiochim. Acta 65, 173-179.

Dinkelaker B., Rohmeld V., Marshneret H. (1989) Citric acid excretion and precipitation of calcium citrate in the rhizosphere of white lupin, Plant Cell. Environ. 12, 285-292.

Hummel W., Glaus M.A., van Loon L.R. (2000) Trace metal-humate interactions. II. The «conservative roof» model and its application, Appl. Geochem. 15, 975-1001.

Hursthouse A.S., Livens F.R. (1993) Evidence for the remobilisation of transuranic elements in the terrestrial environment, Environ. Geochem. Health 15, 163-171.

Keague J., Cheshire M.V., Andreux F., Berthelin J. (1986) Organomineral complexes in relation to pedogenesis: interaction of soil minerals with natural organics and microbes, Soil Sci. Soc. Amer. 17, 549-592.

Kim J.I., Marquardt C.M. (1999) Chemical Reaction of Np(V) with Humic Colloids in Groundwater: Influence of Purification on the Complexation Behaviour, Radiochim. Acta 87, 105-108.

Kim J.I., Rhee D.S., Buckau G., Morgenstern A. (1997) Americium(III)-Humate Interaction in Natural groundwater: Influence of Purification on Complexation Properties, Radiochim. Acta 79, 173182.

Kim J.I., Delakowitz B., Lazik D. (1994) A column experiment for the study of colloidal radionuclide migration in Gorleben aquifer systems, Radiochim. Acta 66-67, 165-182.

Kung K.S., Triay I.R. (1994) Effect of natural organics on Cd and Np sorption, Radiochim. Acta 6667, 421-426.

Labonne-Wall N., Moulin V., Vilarem J.P. (1997) Retention properties of humic substances onto amorphous silica: Consequences for the sorption of cations, Radiochim. Acta. 79, 37-49.

Lefebre-Drouet E., Rousseau-Djarbri M.F., Berthremieux R. (1993) Mobilisation du fer d'un sol en milieu lactique, Sci. Sol 31, 47-58.

Marquardt C., Kim J.I. (1998) Complexation of $\mathrm{Np}(\mathrm{V})$ with Fulvic Acid, Radiochim. Acta 81, 143 148.

Morello M., Colle C., Bernard J. (1986) Mesure du neptunium 237 dans l'environnement, J. Less Com. Metal 122, 569-576.

Moulin V., Reiller P., Dautel C., Plancque G., Laszak I., Moulin C. (1999) Complexation of Eu(III), Th(IV) and $\mathrm{U}(\mathrm{VI})$ by humic substances. In: Effects of humic substances on the migration of radionuclides: complexation and transport of actinides. Second technical progress report, EC F14WCT96-0027, pp. 83-117.

Moulin V., Moulin C. (1995) Fate of actinides in the presence of humic substances under conditions relevant to nuclear waste disposal, Appl. Geochem. 10, 573-580. 


\section{S. ROUSSEL-DEBET et al.}

Nishita H., Wallace A., Romney E. M., Schulz R.K. (1979) Effect of soil type on the extractability of ${ }^{237} \mathrm{~Np},{ }^{239.240} \mathrm{Pu},{ }^{241} \mathrm{Am}$ and ${ }^{244} \mathrm{Cm}$ as a function of $\mathrm{pH}$. NUREG CR-0997, UCLA 12-1192.

Nisbet A.F., Shaw S., Salbu B. (1993) Association of radionuclides with different molecular size fractions in soil solution: implications for plant uptake, J. Environ. Radioactiv. 18, 71-84.

Nitsche H. (1993) Measured solubilities and speciation of Np, Pu and Am in a typical groundwater from the Yucca Mountain Region. Milestone report 3010 WBS 1234131.

Olofsson U., Allard B. (1983) Complexes of actinides with naturally occuring organic substances. Literature survey. SKBF- KBS Sweden Teknisk Rapport 83-09.

Rao L., Choppin G.R., (1995) Thermodynamic study of the complexation of neptunium (V) with humic acids, Radiochim. Acta 69, 87-95

Roussel-Debet S., Colle C., Hurtevent P. (1997) Biodisponibilité des transuraniens dans les sols - I Expérimentations 1997. Rapport IPSN/DPRE/SERE/97/040.

Roussel-Debet S., Colle C., Hurtevent P., Morello M., Claude L. (1998) Influence of organic acids on actinide desorption from cultivated soil. In: 8th annual meeting of Society of Environmental Toxicology and Chemistry, 14-18 avril 1998, Bordeaux, SETAC-Europe, Brussels.

Silva R., Nitsche H. (1995) Actinide Environmental Chemistry, Radiochim. Acta 70-71, 337-396.

Tanaka T., Nagao S., Sakamoto Y., Ohnuki T., Ni S.W., Senoo M. (1997) Distribution coefficient in the sorption of radionuclides onto Ando soil in the presence of humic acid. Influence of the molecular size of humic acid, J. Nucl. Sci. Technol. 34, 829-834.

Thibault D.H., Sheppard M.I., Smith P.A. (1990) A critical compilation and review of default soil solidliquid partition coefficients $K d$, for use in environmental assessments. AECL, ROEILO10125

Tipping E., Woolf C, Kelly M. (1995) Solid-solution distribution of radionuclides in acid soils; application of the WHAM chemical speciation model, Environ. Sci. Technol. 29, 1365-1372.

Watteau F., Berthelin J. (1994) Microbial dissolution of iron and aluminium from soil minerals: efficiency and specificity of hydroxamate siderophores compared to aliphatic acids, Euro. J. Soil Biol. 30, 1-9.

Yong P.M., Macaskie L.E. (1997) Removal of lanthanum, uranium and thorium from the citrate complexes by immobilized cells of citrobacter sp. In: A flow-through reactor: implications for the decontamination of solutions containing plutonium, Biotech. Lett. 19, 251-256. 